\title{
GENETIC VARIABILITY, HERITABILITY AND CORRELATION IN WATERMELON
}

\author{
Abd Rabou, A.M. and E.M. El-Sayd \\ Veg. Res. Depts., Horticulture Res. Inst., Agric. Res. Center, Giza, Egypt. \\ Email-aymanabtrabou40@gmail.com \\ Key words: Genetic Variability, Heritability, Correlation, Watermelon.

\section{ABSTRACT}

This study was conducted at Kaha Vegetable Research Farm (K.V.R.F), Qalubia governorate, Egypt and private farm in Wady ElNitroun during the period from 2015 to 2017 to determine the variability, heritability and correlation among some watermelon inbred lines. The treatments consist of forty two watermelon inbred lines, laid out in randomized complete block design (RCBD) and replicated three times. Inbred lines were evaluated for ten characters to determine variability, estimate heritability and correlation. Analysis of variance revealed highly significant $(\mathrm{P}<0.05)$ for all characters. The results indicated the presence of substantial variability among the genotypes. Genotypic coefficient of variation $(\mathrm{GCV})$, phenotypic coefficient of variation (PCV) and broad Sense heritability (h2) estimates ranges from 14.05-72.98, 14.86-73.84 and 89.3699.94\% respectively. The high estimates of GCV and PCV in this study indicated the existence of variability and selection can be done. Whereas high estimate of $\mathrm{h} 2$ for the tested traits indicated that these characters were highly heritable and selection can be imposed. Significant phenotypic correlation for fruit diameter, fruit length, fruit weight and number of fruits per plant with total yield per plant revealed that, these characters were primarily influenced by their direct contribution to higher yield. Four inbred lines had best marketable yield and fruit quality as red and yellow watermelon inbred lines. Therefore, it is recommended for an effective selection of those characters which could be adopted for cultivar improvement and hybridization program and more research is needed to validate the findings.

\section{INTRODUCTION}

Watermelon (citrullus lanatus thunb.) is one of the most popular and widely grown cucurbitaceous fruit vegetable crops in tropical and subtropical countries of the world. It's global consumption is greater than that of any other cucurbits (Goreta $\boldsymbol{e t}$ al. 2005). In desert areas, the juicy flesh serves as substitute for drinking water. The flesh which is soft and spongy is reddish or pink or yellow or yellowish white in color (Anburani et al. 2019)

Success of watermelon production and profits accrued are determined by the choice of genotype, production method and market demand. Basic market requirements relate primarily to fruit characteristics such as fruit size, rind thickness, taste, meat color, fruit shape and color 
(Jelica et al. 2011). Current demands of both consumers and producers are an important item in watermelon breeding programs, especially for fruit traits. These requirements are: fruit size of 4-6 kg, good taste (8-10\% TSS), rind thickness up to $1.5 \mathrm{~cm}$, round to oval-shaped fruit, small seeds, and very high meat ratio. Watermelon breeding includes the development of breeding lines, cultivars and hybrids using different crossing methods. To increase the efficiency of breeding, it is necessary to know the genetic basis of the traits that are being improved in the available material selection.

Anburani et al (2019) studied the genetic variability and heritability in thirty watermelon genotypes and the results showed significant differences among all studied characters.

Genetic variation among traits is important for a crop improvement programme and in breeding or selecting desirable types. Information of variability patterns allows breeders to comprehend the evolutionary associations among genotypes in a better way, to collect genotypes in a more organized manner, and to make plan to incorporate valuable material in their germplasm (Bretting and Widrlechner,1995). Anburani et al. (2019) reported that characters, viz., fruits diameter, , number of fruits per plant and yield per plant, recorded high estimate of PCV and moderate estimation of GCV. Meanwhile, the characters, viz., number of seeds per fruits, and fruit diameter recorded moderate estimate of PCV and GCV. Also, high heritability (broad sense) was observed for 100 seed weight, number of seeds per fruit, fruit diameter, fruit length, yield per plant, and number of fruits per plant.

Hence, the present investigation was attempted to find out the extent of genotypic variability and heritability for yield and yield components with a view to identify the best red and yellow watermelon inbred lines.

\section{MATERIALS AND METHODS}

This study was conducted during the three successive summer seasons from 2015 to 2017.Forty two watermelon inbred lines (Table 1) collected from diverse sources (40 from Netherland Gene Bank and 2 from Philippine Agr. Co.). Selfed pollinated at Kaha Vegetable Research Farm (K.V.R.F), Qalubia governorate, Egypt were done for 7 generations to have pure inbred lines. These inbred lines cultivated at K.V.R.F in 2015 to ensure their purity. Forty two watermelon inbred lines cultivated at private farm at Wady El- Nitroun in summer seasons of 2016 and 2017 to estimate phenotypic coefficient of variation (PVC), genotypic coefficient of variation (GCV) and heritability for yield, yield components and fruit quality. Seedlings were transplanted on March $1^{\text {st }}, 2016$ and March $2^{\text {nd }}$ 2017in the open field. The experiment was set up in a randomized complete block design with three replications. The experiment unit area was $20 \mathrm{~m}^{2}$. Twelve watermelon plants were planted in each unit: three rows $2 \mathrm{~m}$ apart, and plants spacing in row, $1 \mathrm{~m}$. Land preparation, fertilizer application and other field practices were conducted according to recommendations of the Egyptian Ministry of Agriculture. 
Table 1. performance of studied watermelon genotypes.

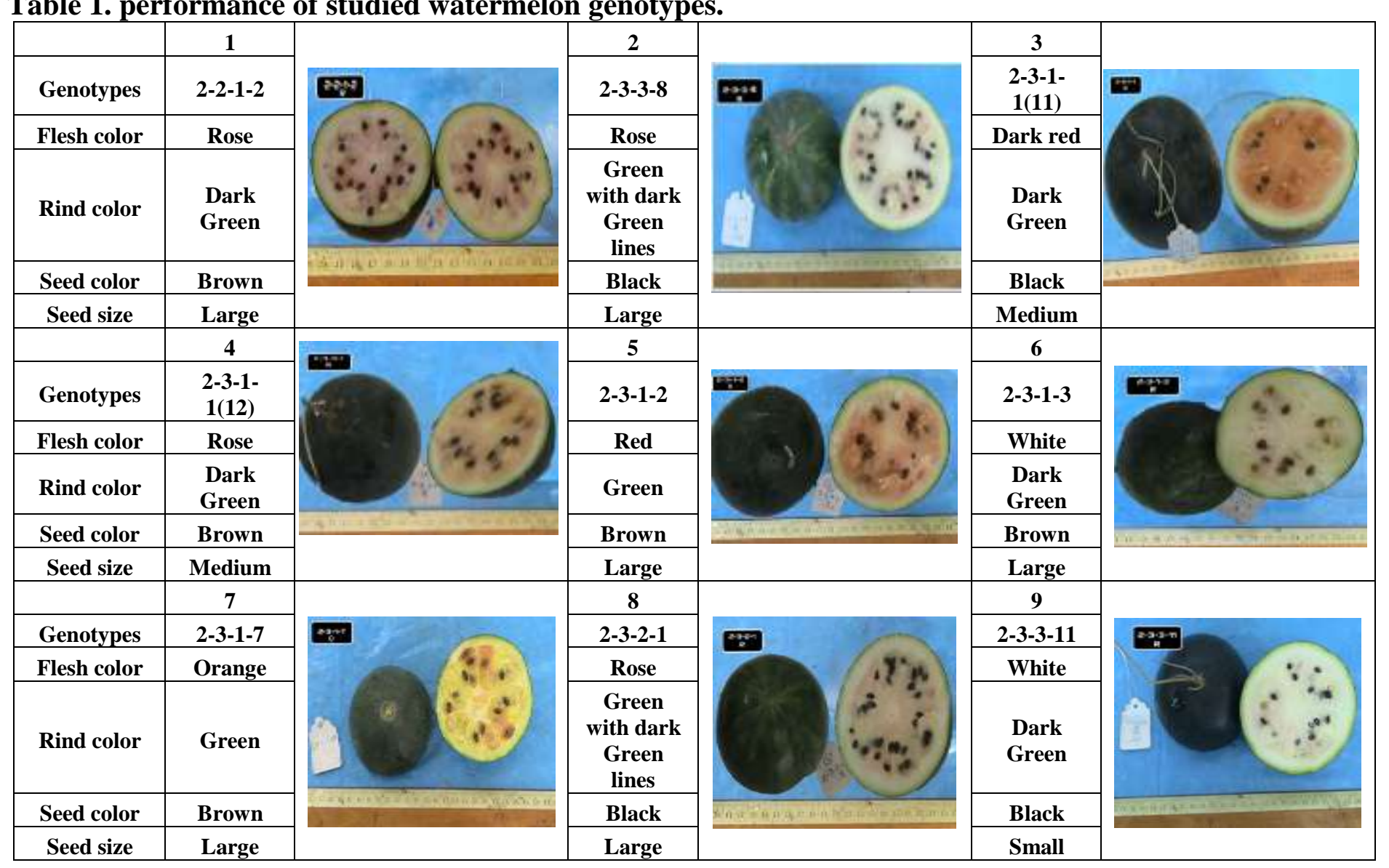


Table 1. Count

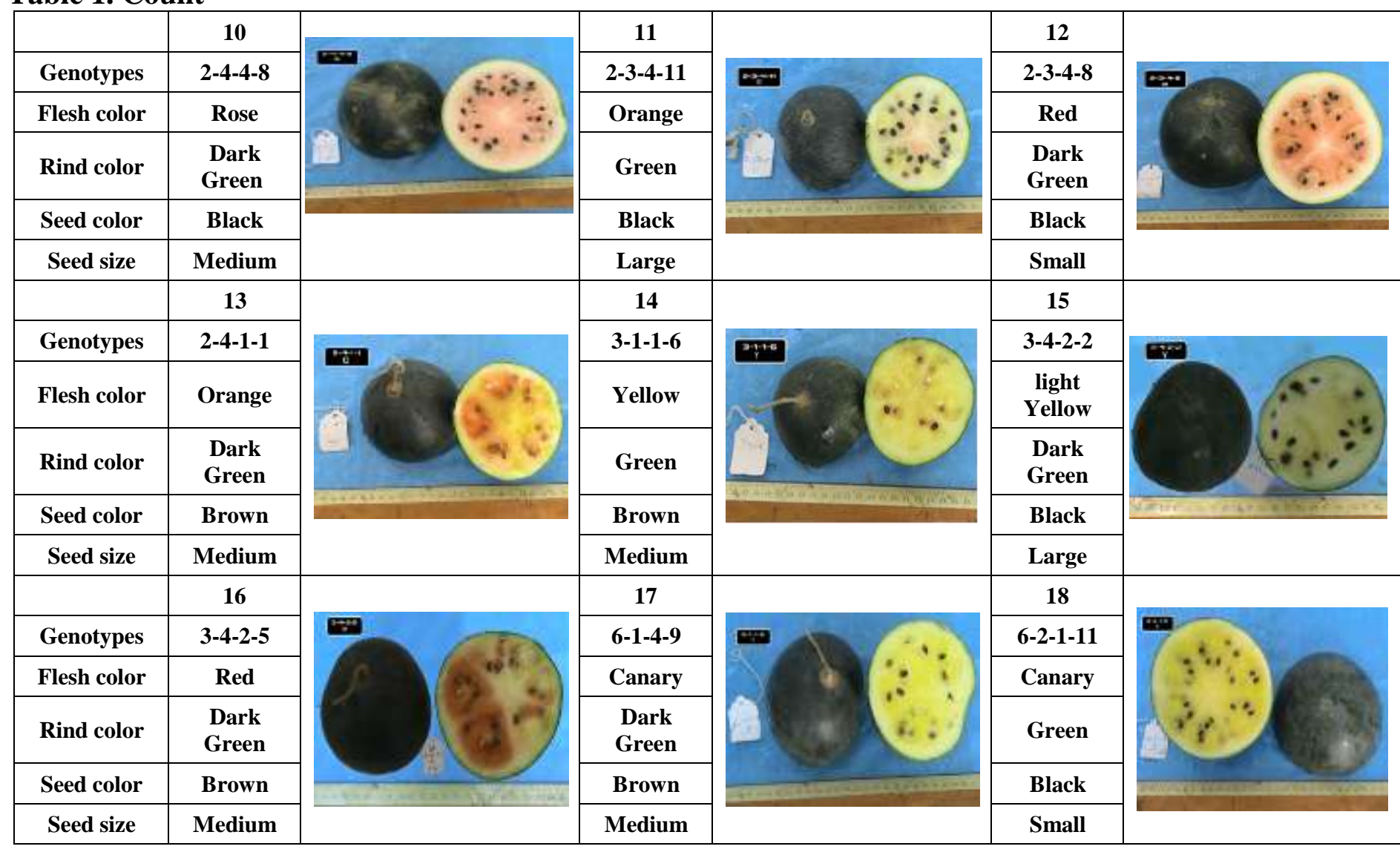

s

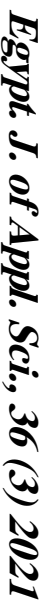


Table 1. Count

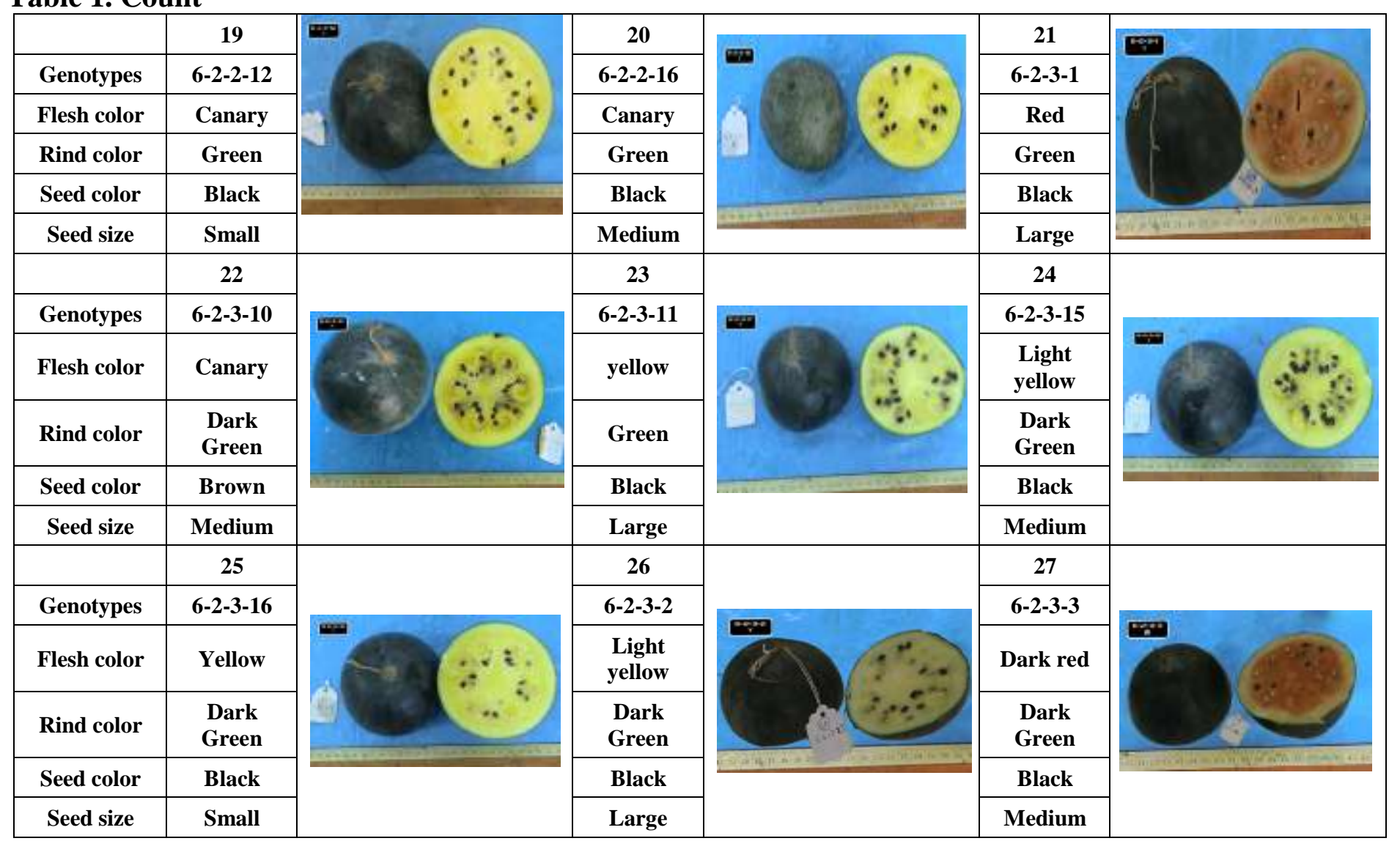


Table 1. Count

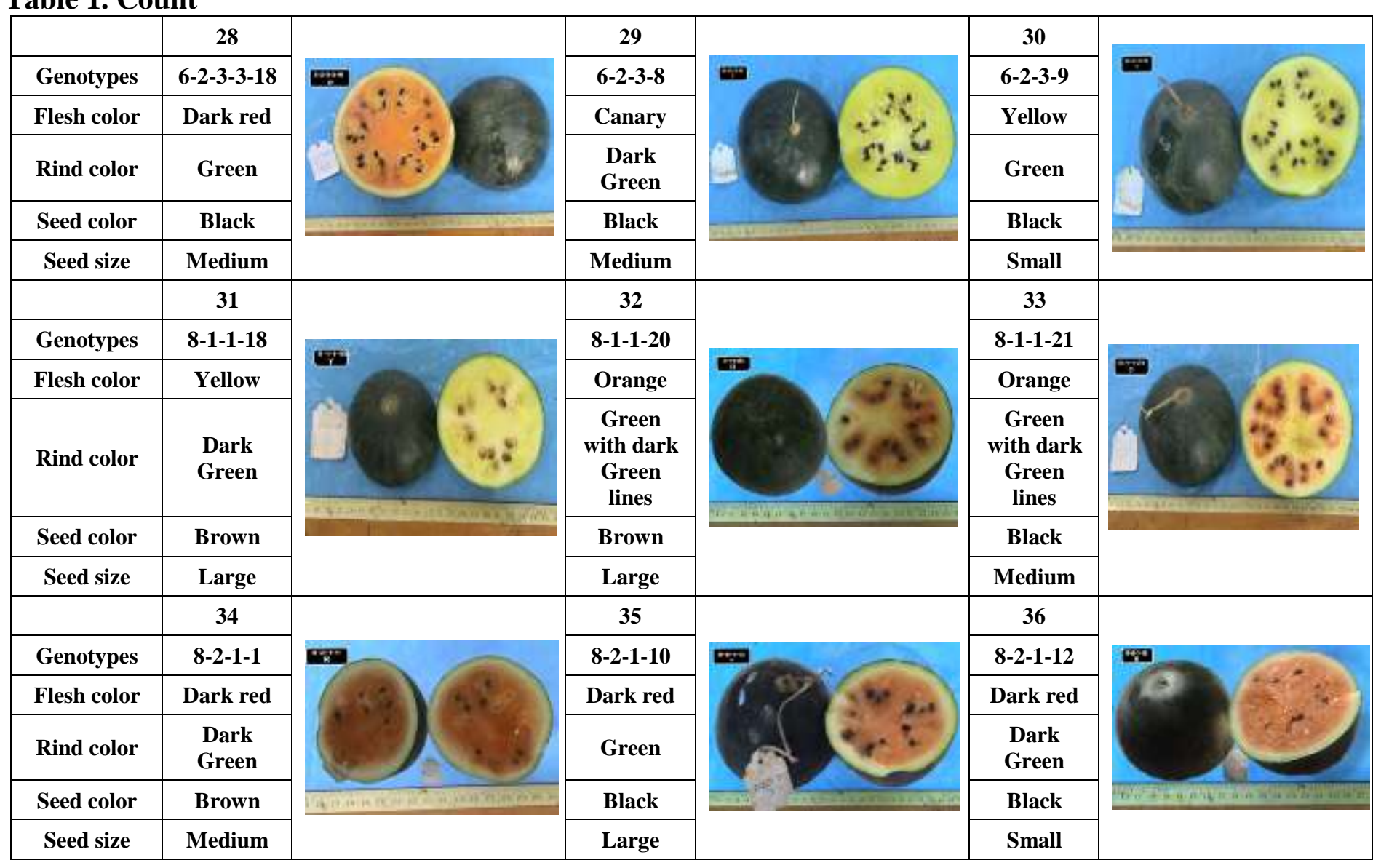

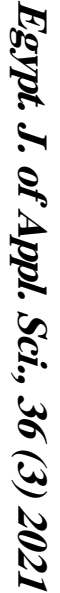


Table 1. Count

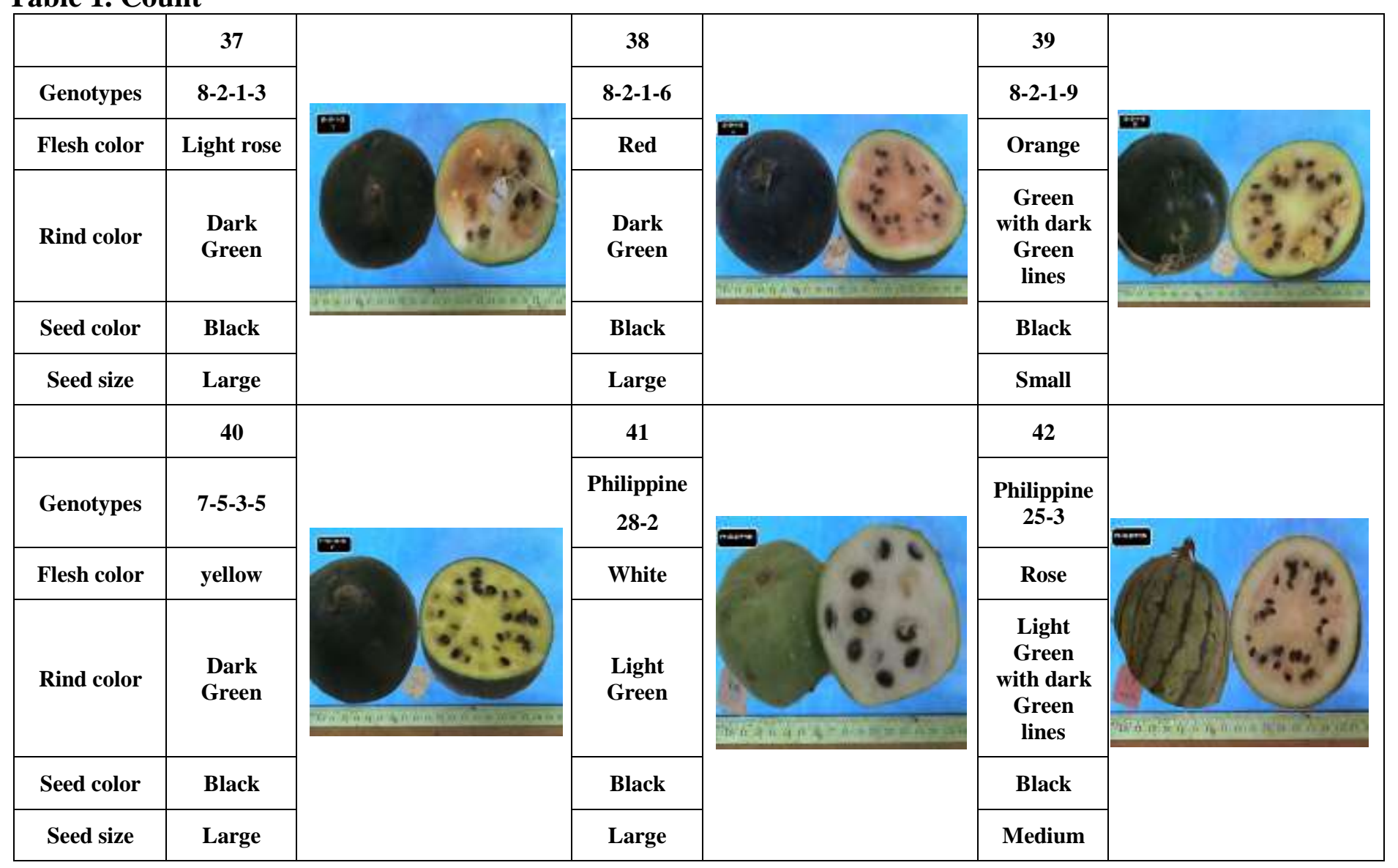


Studied watermelon genotypes were recorded for 10 quantities traits, viz., number of fruits per plant (NF), average fruit weight $(\mathrm{kg})$ $(\mathrm{FW})$, total yield per plant $(\mathrm{kg})(\mathrm{TY})$, rind thickness $(\mathrm{cm})(\mathrm{RT})$, total soluble solids (\%) (TSS), fruit length $(\mathrm{cm})(\mathrm{FL})$, fruit diameter $(\mathrm{cm})$ (FD), number of seeds per fruit (NS), 100 seeds weight (g) (SW), and fruit shape index (SHI)).

Mujaju (2009) reported that the pulp of watermelon varies from yellow or green (wild forms) to dark red (cultivars).Soluble solids were measured in degrees brix using a refractometer that was dipped three times into the flesh in the center of the fruit (Gusmini and Wehner, 2005).

Observations were recorded on various characters and subjected to statistical analysis. Collected data were subjected to analysis of variance (ANOVA) using Statistix 8software version 8.0 (2003) and correlation was also determined. Genotypic coefficient of variation (GCV), phenotypic coefficient of variation (PCV), and environmental coefficient of variation (ECV) were calculated according to Sivasubramanian and Menan(1973) and heritability $\left(\mathrm{h}^{2}\right)$ was calculated according to Johnson et al(1955).

\section{RESULTS AND DISCUSSION}

The general analysis of variance showed significant differences among the 42 genotypes of watermelon for all ten studied characters in 2016 and 2017 (Table 2).

\section{1- Number of fruits per plant:}

Data showed that only line 6-2-3-16 and 6-2-3-15 had the highest values of number of fruits per plant in both two seasons (7.0, 6.6, 6.7 and 6.2 , respectively), with significant differences with the other inbred lines in 2016 and 2017. The lowest number of fruits per plant was ranged from 2.8 to 3.2 and obtained at 5 inbred lines in first season and 4 inbred lines in second season with significant differences with the other inbred lines.

\section{2-Fruit weight:}

Data obtained on fruit weight are illustrated in Table (2).It is clear that the genotype6-2-2-16 gave the heaviest fruit weight in both two seasons ( 8.2 and $8.1 \mathrm{~kg}$, respectively). The lightest fruit weight was obtained in genotype 2-4-1-1 (1.9 and $2.0 \mathrm{~kg})$ in both seasons respectively. it could be concluded that genotype 6-2-2-16had the highest value of fruit length, diameter and weight. (Gusmini and Wehner 2005) studied 80 cultivars and found the range of mean value revealed sufficient variation for average watermelon fruit weight between 1.59 to 9.58 . 


\section{3- Total yield/plant:}

Data obtained on the total yield per plant are illustrated in Table (2). The results indicated that evaluation of genotypes based on higher yield per plant ranged from $(7.6$ to $40.5 \mathrm{~kg})$ and $(8$ to $42.5 \mathrm{Kg})$ in both seasons respectively, genotype8-2-1-12 $(40.50$ and $42.5 \mathrm{Kg})$ as the top ranking genotype with significant differences with other inbred lines. Philippine 28-2 had the lowest values of total yield per plant in two season without significant differences with 4 inbred lines in 2016 and 7 inbred lines in 2017.

\section{4- Rind thickness:}

Rind thickness in the studied 42 watermelon inbred lines studied ranged from 0.34 to $2.0 \mathrm{~cm}$ (Table 2) in the two summer seasons of 2016 and 2017. Rind thickness was significantly greatest in the genotype6-2-38 in both seasons of study (2.00 and $1.98 \mathrm{~cm}$, respectively). Slim rind were obtained with Philippine 28-2 in both seasons $(0.4$ and $0.34 \mathrm{~cm})$ respectively. Gusmini et al. (2004) divided watermelons into three groups based on the rind thickness. The first group were genotypes with the rind thickness $>1.9 \mathrm{~cm}$, the second from 1.0 to $1.9 \mathrm{~cm}$ and the third $<1,0 \mathrm{~cm}$.

\section{5- Total soluble solids (\%):}

Total soluble solids in the studied 42 watermelon inbred lines in both seasons had wide ranged from $3.0 \%$ to 12.4 (Table 2). In both seasons, total soluble solids was significantly greatest in 6-2-3-16 in both seasons of study (12.3 and $12.4 \%)$ respectively. LowestTSS was obtained with Philippine 28-2 in both seasons (3 and $3 \%$, respectively). Jelicaet al. (2011) studied six parents and their 30 hybrids for sugar content which ranged from 8.8 to $10.8 \%$.

\section{6-Fruit length}

Data obtained on the trait fruit length are illustrated in table (2). The results obtained that the 4 inbred lines (2-3-1-1-11, 2-3-3-11, 6-2-216 and 8-2-1-12) gave fruit length above $40 \mathrm{~cm}$ in both two seasons. On the contrary, 2-4-1-1gave the shortest fruit length on both season (18.8 and $17.7 \mathrm{~cm}$, respectively). These results agreement with Gusmini and Wehner(2005) who studied 80watermelon cultivars and found that fruit length ranged from 20.17 to $52.00 \mathrm{~cm}$.

\section{7-Fruit diameter}

Data obtained on the trait fruit diameter of 42 watermelon inbred lines are presented in table (2). Data showed that only line 6-2-2-16gave the highest values of fruit diameter in both seasons $(39.5$ and $38.2 \mathrm{~cm}$, respectively), with significant differences with the other inbred lines in 2016 and 2017. The lowest fruit diameter was obtained on2-4-1-1 (12.6 and $13.0 \mathrm{~cm}$ ) in both seasons, respectively. 
Table 2. Means performance of studied watermelon genotypes.

\begin{tabular}{|c|c|c|c|c|c|c|c|c|c|c|c|c|c|c|c|c|c|c|c|c|c|}
\hline & & \multicolumn{2}{|c|}{$\mathbf{N F}$} & \multicolumn{2}{|c|}{ FW } & \multicolumn{2}{|c|}{ TY } & \multicolumn{2}{|c|}{ RT } & \multicolumn{2}{|c|}{ TSS } & \multicolumn{2}{|c|}{ FL } & \multicolumn{2}{|c|}{ FD } & \multicolumn{2}{|c|}{ NS } & \multicolumn{2}{|c|}{ SW } & \multicolumn{2}{|c|}{ SHI } \\
\hline & enotypes & 2016 & 2017 & 2016 & \begin{tabular}{|l|}
2017 \\
\end{tabular} & 2016 & 2017 & 2016 & 2017 & 2016 & 2017 & 2016 & 2017 & 2016 & 2017 & 2016 & \begin{tabular}{|l|}
2017 \\
\end{tabular} & 2016 & 2017 & 2016 & 2017 \\
\hline 1 & 2-2-1-2 & $\begin{array}{l}4.5 \\
\end{array}$ & 4.1 & 3.3 & 3.2 & \begin{tabular}{|l|l|}
13.5 \\
\end{tabular} & \begin{tabular}{|l|}
13.1 \\
\end{tabular} & 0.64 & 0.69 & 10.0 & 10.0 & \begin{tabular}{|l|}
28.1 \\
\end{tabular} & 30.5 & 20.2 & 21.7 & 291.1 & \begin{tabular}{|l|}
294.6 \\
\end{tabular} & 14.6 & 15.2 & 1.40 & 1.41 \\
\hline 2 & 2-3-3-8 & 3.2 & 3.0 & 4.9 & 4.8 & \begin{tabular}{|l|l|}
15.1 \\
\end{tabular} & \begin{tabular}{|l|}
14.4 \\
\end{tabular} & 1.30 & 1.32 & \begin{tabular}{|l|}
8.1 \\
\end{tabular} & 8.1 & 32.9 & 36.7 & 32.2 & 31.0 & 314.1 & \begin{tabular}{|l|l|}
309.7 \\
\end{tabular} & \begin{tabular}{|l|}
7.4 \\
\end{tabular} & 8.0 & 1.10 & 1.18 \\
\hline 3 & 2-3-1-1(11) & 4.4 & 4.8 & 6.4 & 6.2 & 30.3 & \begin{tabular}{|l|}
29.8 \\
\end{tabular} & 1.19 & 1.11 & 11.0 & 10.7 & \begin{tabular}{|l|l}
43.7 \\
\end{tabular} & 41.3 & \begin{tabular}{|l|l|}
34.8 \\
\end{tabular} & 33.5 & 156.0 & \begin{tabular}{|l|}
150.0 \\
\end{tabular} & \begin{tabular}{|l|l|}
7.7 \\
\end{tabular} & 8.3 & 1.23 & 1.23 \\
\hline 4 & 2-3-1-1(12) & \begin{tabular}{ll|}
4.8 \\
\end{tabular} & 4.3 & 4.6 & 4.8 & \begin{tabular}{|l|l|}
21.9 \\
\end{tabular} & 20.5 & 1.00 & 0.97 & \begin{tabular}{|l|}
9.0 \\
\end{tabular} & \begin{tabular}{|l|}
9.0 \\
\end{tabular} & 32.9 & 35.6 & \begin{tabular}{|l|l|}
29.7 \\
\end{tabular} & 29.2 & 351.0 & 354.5 & \begin{tabular}{|l|l|}
7.3 \\
\end{tabular} & 7.9 & 1.17 & 1.22 \\
\hline 5 & 2-3-1-2 & 4.5 & 4.1 & 4.0 & 4.1 & 18.0 & \begin{tabular}{|l|}
16.7 \\
\end{tabular} & 1.57 & 1.52 & 5.5 & 5.5 & 32.1 & 34.5 & 22.0 & 23.9 & 435.5 & 429.5 & 10.8 & 10.3 & 1.47 & 1.44 \\
\hline 6 & $2-3-1-3$ & 4.9 & 5.3 & 3.0 & 2.8 & \begin{tabular}{|l|l|}
14.4 \\
\end{tabular} & \begin{tabular}{|l|}
14.7 \\
\end{tabular} & 1.00 & 0.97 & 8.6 & 8.7 & \begin{tabular}{|l|}
29.6 \\
\end{tabular} & 28.2 & \begin{tabular}{|l|l|}
20.9 \\
\end{tabular} & 20.2 & 265.5 & \begin{tabular}{|l|}
269.0 \\
\end{tabular} & \begin{tabular}{|l|}
5.6 \\
\end{tabular} & 5.1 & 1.40 & 1.40 \\
\hline 7 & 2-3-1-7 & 4.5 & 4.1 & 4.9 & 4.8 & 22.3 & \begin{tabular}{|l|}
19.6 \\
\end{tabular} & 1.00 & 0.98 & 8.1 & 8.1 & \begin{tabular}{|l|}
33.2 \\
\end{tabular} & 35.6 & 29.9 & 28.6 & 168.1 & \begin{tabular}{|l|l|}
163.7 \\
\end{tabular} & \begin{tabular}{|l|}
5.9 \\
\end{tabular} & 6.4 & 1.17 & 1.24 \\
\hline 8 & 2-3-2-1 & 4.2 & 3.7 & 5.1 & 5.3 & 21.4 & \begin{tabular}{|l|}
19.2 \\
\end{tabular} & 1.60 & 1.58 & \begin{tabular}{|l|l|}
9.8 \\
\end{tabular} & \begin{tabular}{|l|l|}
9.8 \\
\end{tabular} & \begin{tabular}{|l|l|}
37.1 \\
\end{tabular} & 39.5 & \begin{tabular}{|l|l|l}
33.7 \\
\end{tabular} & 32.4 & 298.9 & \begin{tabular}{|l|}
292.9 \\
\end{tabular} & \begin{tabular}{|l|l|}
4.9 \\
\end{tabular} & $\begin{array}{ll}4.4 \\
\end{array}$ & 1.17 & 1.22 \\
\hline 9 & 2-3-3-11 & 3.9 & 4.3 & 6.9 & 7.0 & 26.6 & \begin{tabular}{|l|}
29.8 \\
\end{tabular} & 1.70 & 1.67 & \begin{tabular}{|l|l|}
8.7 \\
\end{tabular} & \begin{tabular}{|l|l|}
8.8 \\
\end{tabular} & \begin{tabular}{|l|l|}
42.4 \\
\end{tabular} & 41.3 & \begin{tabular}{|l|}
35.9 \\
\end{tabular} & 34.6 & 211.4 & \begin{tabular}{|l|}
214.9 \\
\end{tabular} & \begin{tabular}{|l|}
6.1 \\
\end{tabular} & 6.7 & 1.20 & 1.19 \\
\hline 10 & $2-4-4-8$ & 4.2 & 3.8 & 4.0 & 3.8 & 16.7 & \begin{tabular}{|l|}
14.4 \\
\end{tabular} & 1.60 & 1.62 & 7.2 & 7.3 & \begin{tabular}{|l|}
31.5 \\
\end{tabular} & 33.9 & \begin{tabular}{|l|}
24.5 \\
\end{tabular} & 23.2 & 131.8 & \begin{tabular}{|l|}
127.3 \\
\end{tabular} & \begin{tabular}{|l|}
4.9 \\
\end{tabular} & 4.4 & 1.40 & 1.46 \\
\hline 11 & 2-3-4-11 & 4.5 & 4.0 & 5.8 & 6.0 & \begin{tabular}{|l|}
26.5 \\
\end{tabular} & \begin{tabular}{|l|}
23.9 \\
\end{tabular} & 1.20 & 1.18 & \begin{tabular}{|l|}
6.9 \\
\end{tabular} & \begin{tabular}{|l|}
6.9 \\
\end{tabular} & \begin{tabular}{|l|}
38.7 \\
\end{tabular} & 41.1 & \begin{tabular}{|l|}
34.6 \\
\end{tabular} & 33.3 & 36.4 & \begin{tabular}{|l|}
37.8 \\
\end{tabular} & \begin{tabular}{|l|}
6.9 \\
\end{tabular} & 7.4 & 1.17 & 1.23 \\
\hline 12 & $2-3-4-8$ & 5.5 & 5.9 & 3.3 & 3.5 & \begin{tabular}{|l|}
18.3 \\
\end{tabular} & \begin{tabular}{|l|}
20.6 \\
\end{tabular} & 1.50 & 1.47 & 8.9 & 8.9 & \begin{tabular}{|l|}
36.0 \\
\end{tabular} & 31.9 & 24.2 & 22.9 & 268.8 & \begin{tabular}{|l|}
264.3 \\
\end{tabular} & \begin{tabular}{|l|}
6.0 \\
\end{tabular} & 6.5 & 1.43 & 1.39 \\
\hline 13 & 2-4-1-1 & 4.5 & 3.8 & 1.9 & 2.0 & 8.1 & 7.6 & 1.40 & \begin{tabular}{|l|l|}
1.38 \\
\end{tabular} & \begin{tabular}{|l|}
6.3 \\
\end{tabular} & \begin{tabular}{|l|}
6.4 \\
\end{tabular} & \begin{tabular}{|l|l|}
18.8 \\
\end{tabular} & 17.7 & 12.6 & 13.0 & 327.8 & \begin{tabular}{|l|}
321.8 \\
\end{tabular} & 3.0 & 3.1 & 1.43 & 1.36 \\
\hline 14 & $3-1-1-6$ & 4.2 & 4.0 & 5.2 & 5.0 & \begin{tabular}{|l|}
23.4 \\
\end{tabular} & \begin{tabular}{|l|}
20.1 \\
\end{tabular} & 1.90 & $\begin{array}{l}1.87 \\
\end{array}$ & 11.6 & 11.2 & \begin{tabular}{|l|}
34.6 \\
\end{tabular} & $\begin{array}{l}37.0 \\
\end{array}$ & 32.6 & 31.3 & 376.1 & \begin{tabular}{|l|}
370.2 \\
\end{tabular} & \begin{tabular}{|l|}
8.6 \\
\end{tabular} & 9.2 & 1.13 & 1.18 \\
\hline 15 & $3-4-2-2$ & 3.9 & 4.3 & 4.4 & 4.5 & 17.0 & \begin{tabular}{|l|}
19.4 \\
\end{tabular} & 1.20 & 1.22 & 11.2 & 11.0 & \begin{tabular}{|l|}
32.6 \\
\end{tabular} & 35.2 & \begin{tabular}{|l|}
28.9 \\
\end{tabular} & 27.6 & 303.9 & \begin{tabular}{|l|}
298.0 \\
\end{tabular} & \begin{tabular}{|l|}
10.6 \\
\end{tabular} & 11.0 & 1.20 & 1.28 \\
\hline 16 & $3-4-2-5$ & 4.2 & 3.8 & 2.2 & 2.3 & \begin{tabular}{|l|l|}
9.2 \\
\end{tabular} & 8.5 & 1.10 & 1.07 & \begin{tabular}{|l|}
8.8 \\
\end{tabular} & \begin{tabular}{|l|}
8.5 \\
\end{tabular} & \begin{tabular}{|l|}
29.0 \\
\end{tabular} & 25.3 & \begin{tabular}{|l|}
19.6 \\
\end{tabular} & 18.3 & 388.9 & \begin{tabular}{|l|}
392.4 \\
\end{tabular} & \begin{tabular}{|l|}
4.3 \\
\end{tabular} & 3.9 & 1.43 & 1.38 \\
\hline 17 & 6-1-4-9 & 5.4 & 4.8 & 2.7 & 2.5 & \begin{tabular}{|l|}
14.5 \\
\end{tabular} & \begin{tabular}{|l|}
12.4 \\
\end{tabular} & 1.40 & 1.42 & 11.0 & \begin{tabular}{|l|}
10.8 \\
\end{tabular} & \begin{tabular}{|l|}
23.7 \\
\end{tabular} & 26.1 & \begin{tabular}{|l|}
20.4 \\
\end{tabular} & 19.1 & 198.6 & \begin{tabular}{|l|}
192.6 \\
\end{tabular} & \begin{tabular}{|l|l|}
11.1 \\
\end{tabular} & 11.6 & 1.30 & 1.37 \\
\hline 18 & 6-2-1-11 & 5.4 & 4.9 & 3.3 & 3.4 & \begin{tabular}{|l|l|}
17.7 \\
\end{tabular} & \begin{tabular}{|l|}
16.7 \\
\end{tabular} & 0.60 & 0.58 & 9.5 & \begin{tabular}{|c|}
9.6 \\
\end{tabular} & \begin{tabular}{|l|}
28.9 \\
\end{tabular} & 31.3 & \begin{tabular}{|l|}
24.1 \\
\end{tabular} & 22.8 & 267.7 & \begin{tabular}{|l|}
263.2 \\
\end{tabular} & 8.3 & 8.9 & 1.30 & 1.37 \\
\hline 19 & 6-2-2-12 & 6.0 & 6.4 & 4.3 & 4.5 & \begin{tabular}{|l|}
26.2 \\
\end{tabular} & \begin{tabular}{|l|}
28.9 \\
\end{tabular} & 0.50 & \begin{tabular}{|l|l|}
0.48 \\
\end{tabular} & \begin{tabular}{|l|}
6.9 \\
\end{tabular} & \begin{tabular}{|l|}
7.0 \\
\end{tabular} & \begin{tabular}{|l|}
32.4 \\
\end{tabular} & \begin{tabular}{|l|l|}
34.8 \\
\end{tabular} & \begin{tabular}{|l|}
28.6 \\
\end{tabular} & 27.3 & 152.8 & \begin{tabular}{|l|}
148.3 \\
\end{tabular} & \begin{tabular}{|l|}
5.9 \\
\end{tabular} & $\begin{array}{l}6.5 \\
\end{array}$ & 1.20 & 1.27 \\
\hline 20 & 6-2-2-16 & 6.4 & 3.3 & 8.2 & 8.1 & \begin{tabular}{|l|}
1.8 \\
\end{tabular} & \begin{tabular}{|l|}
26.9 \\
\end{tabular} & 1.00 & 0.98 & 11.8 & 11.8 & 40.3 & 42.1 & 39.5 & 38.2 & 363.2 & \begin{tabular}{|l|}
358.7 \\
\end{tabular} & \begin{tabular}{|l}
7.7 \\
\end{tabular} & 7.2 & 1.07 & 1.10 \\
\hline 21 & 6-2-3-1 & 3.9 & 5.8 & 2.6 & 2.7 & 16.7 & \begin{tabular}{|l|}
15.9 \\
\end{tabular} & 1.60 & 1.57 & 8.0 & 8.9 & \begin{tabular}{|l|}
24.1 \\
\end{tabular} & 26.5 & \begin{tabular}{|l|}
20.9 \\
\end{tabular} & 19.6 & 399.6 & \begin{tabular}{|l|}
393.6 \\
\end{tabular} & \begin{tabular}{|l|}
6.5 \\
\end{tabular} & 7.0 & 1.30 & 1.35 \\
\hline 22 & 6-2-3-10 & 4.5 & 4.1 & 3.2 & 3.3 & \begin{tabular}{|l|l|}
14.5 \\
\end{tabular} & \begin{tabular}{|l|}
13.6 \\
\end{tabular} & 1.40 & 1.38 & \begin{tabular}{|l|}
9.4 \\
\end{tabular} & \begin{tabular}{|l|} 
\\
\end{tabular} & \begin{tabular}{|l|}
28.4 \\
\end{tabular} & 30.8 & \begin{tabular}{|l|}
24.7 \\
\end{tabular} & 22.0 & 393.3 & \begin{tabular}{|l|}
396.8 \\
\end{tabular} & \begin{tabular}{|l|}
10.5 \\
\end{tabular} & 10.0 & 1.27 & 1.40 \\
\hline 23 & 6-2-3-11 & 4.5 & 4.9 & 4.4 & 4.5 & \begin{tabular}{|l|l|}
19.7 \\
\end{tabular} & \begin{tabular}{|l|}
22.2 \\
\end{tabular} & 1.30 & 1.27 & \begin{tabular}{|l|}
5.8 \\
\end{tabular} & \begin{tabular}{|l|}
5.8 \\
\end{tabular} & \begin{tabular}{|l|}
32.3 \\
\end{tabular} & 35.1 & 28.6 & 27.3 & 308.3 & \begin{tabular}{|l|}
303.8 \\
\end{tabular} & \begin{tabular}{|l|}
8.9 \\
\end{tabular} & 9.4 & 1.20 & 1.29 \\
\hline 24 & 6-2-3-15 & 6.7 & 6.2 & 3.8 & 4.0 & 25.5 & \begin{tabular}{|l|}
24.3 \\
\end{tabular} & 1.20 & 1.22 & 11.9 & 11.8 & \begin{tabular}{|l|l}
31.8 \\
\end{tabular} & 34.2 & 25.0 & 23.7 & 152.0 & 155.5 & \begin{tabular}{|l|l|}
11.1 \\
\end{tabular} & 11.6 & 1.37 & 1.44 \\
\hline 25 & 6-2-3-16 & 7.0 & 6.6 & 4.5 & 4.6 & \begin{tabular}{|l|l|}
31.6 \\
\end{tabular} & \begin{tabular}{|l|}
30.3 \\
\end{tabular} & 0.70 & 0.67 & 12.3 & 12.4 & \begin{tabular}{|l|l|}
34.7 \\
\end{tabular} & 35.5 & 29.4 & 28.1 & 35.8 & \begin{tabular}{|l|}
39.3 \\
\end{tabular} & \begin{tabular}{|l|}
5.9 \\
\end{tabular} & 6.5 & 1.23 & 1.26 \\
\hline 26 & 6-2-3-2 & 4.2 & 4.6 & 4.1 & 4.2 & \begin{tabular}{|l|l|}
17.3 \\
\end{tabular} & \begin{tabular}{|l|l|}
19.4 \\
\end{tabular} & 1.30 & 1.32 & 8.9 & 8.9 & \begin{tabular}{|l|l|}
37.7 \\
\end{tabular} & 34.6 & 27.1 & 25.8 & 251.3 & \begin{tabular}{|l|l|}
254.8 \\
\end{tabular} & \begin{tabular}{|l|l|}
3.4 \\
\end{tabular} & 3.2 & 1.37 & 1.34 \\
\hline 27 & 6-2-3-3 & 3.2 & 2.8 & 2.9 & 2.7 & 9.3 & 7.6 & 1.10 & 1.07 & 3.9 & 3.9 & \begin{tabular}{|l|}
24.2 \\
\end{tabular} & 26.6 & 21.3 & 19.9 & 200.8 & \begin{tabular}{|l|}
196.3 \\
\end{tabular} & \begin{tabular}{|l|l|}
9.4 \\
\end{tabular} & 10.0 & 1.20 & 1.34 \\
\hline 28 & \begin{tabular}{|l|}
$6-2-3-3-18$ \\
\end{tabular} & 5.1 & 4.6 & 5.9 & 6.0 & \begin{tabular}{|l|l|}
29.9 \\
\end{tabular} & \begin{tabular}{|l|}
27.2 \\
\end{tabular} & 1.20 & 1.18 & 10.5 & 10.3 & \begin{tabular}{|l|l|}
36.9 \\
\end{tabular} & 39.8 & \begin{tabular}{|l|l|}
34.1 \\
\end{tabular} & 32.8 & 193.5 & \begin{tabular}{|l|l|}
190.7 \\
\end{tabular} & \begin{tabular}{|l|}
9.1 \\
\end{tabular} & 8.6 & 1.13 & 1.21 \\
\hline 29 & 6-2-3-8 & 3.9 & 4.3 & 5.1 & 5.2 & 19.6 & \begin{tabular}{|l|}
22.3 \\
\end{tabular} & 2.00 & 1.98 & \begin{tabular}{|l|}
6.8 \\
\end{tabular} & 6.9 & \begin{tabular}{|l|l|}
36.9 \\
\end{tabular} & 39.3 & \begin{tabular}{|l|l|}
33.6 \\
\end{tabular} & 32.3 & 231.4 & \begin{tabular}{|l|}
224.9 \\
\end{tabular} & \begin{tabular}{|l|}
4.6 \\
\end{tabular} & 5.1 & 1.17 & 1.22 \\
\hline 30 & 6-2-3-9 & 2.9 & 3.3 & 4.3 & 4.4 & \begin{tabular}{|l|l|}
12.4 \\
\end{tabular} & \begin{tabular}{|l|l|}
14.5 \\
\end{tabular} & 1.30 & 1.27 & \begin{tabular}{|l|}
7.9 \\
\end{tabular} & \begin{tabular}{|l|}
7.9 \\
\end{tabular} & 32.2 & 34.6 & 27.9 & 26.6 & 280.5 & \begin{tabular}{|l|}
284.0 \\
\end{tabular} & \begin{tabular}{|l|}
8.9 \\
\end{tabular} & 9.4 & 1.23 & 1.30 \\
\hline 31 & $8-1-1-18$ & 3.0 & 2.8 & 3.3 & 3.2 & \begin{tabular}{|l|l|}
10.7 \\
\end{tabular} & 8.8 & 1.50 & 1.48 & 5.8 & \begin{tabular}{|l|l|}
5.8 \\
\end{tabular} & \begin{tabular}{|l|l|}
33.4 \\
\end{tabular} & 30.1 & 22.6 & 21.5 & 165.6 & \begin{tabular}{|l|l|}
161.1 \\
\end{tabular} & \begin{tabular}{|l|}
8.9 \\
\end{tabular} & 9.5 & 1.43 & 1.40 \\
\hline
\end{tabular}


Table 2. Cont.

\begin{tabular}{|c|c|c|c|c|c|c|c|c|c|c|c|c|c|c|c|c|c|c|c|c|c|}
\hline & & \multicolumn{2}{|c|}{$\mathbf{N F}$} & \multicolumn{2}{|c|}{ FW } & \multicolumn{2}{|c|}{ TY } & \multicolumn{2}{|c|}{ RT } & \multicolumn{2}{|c|}{ TSS } & \multicolumn{2}{|c|}{ FL } & \multicolumn{2}{|c|}{ FD } & \multicolumn{2}{|c|}{ NS } & \multicolumn{2}{|c|}{ Sw } & \multicolumn{2}{|c|}{ SHI } \\
\hline \multicolumn{2}{|c|}{ Genotypes } & 2016 & 2017 & 2016 & 2017 & 2016 & 2017 & 2016 & 2017 & 2016 & 2017 & 2016 & 2017 & 2016 & 2017 & 2016 & 2017 & 2016 & 2017 & 2016 & 2017 \\
\hline 32 & $8-1-1-20$ & 3.5 & 3.1 & 5.1 & 5.2 & 18.2 & 16.2 & 1.40 & 1.38 & 10.5 & 10.3 & 36.4 & 38.8 & 33.2 & 31.9 & 386.8 & 382.3 & 8.0 & 8.5 & 1.17 & 1.22 \\
\hline 33 & $8-1-1-21$ & 3.5 & 3.9 & 2.2 & 2.3 & 7.6 & 8.9 & 1.20 & 1.22 & 11.8 & 11.6 & 22.9 & 25.3 & 19.3 & 18.0 & 376.0 & 370.0 & 13.9 & 13.4 & 1.30 & 1.41 \\
\hline 34 & $8-2-1-1$ & 3.9 & 3.3 & 2.8 & 2.7 & 10.9 & 9.0 & 1.30 & 1.32 & 9.0 & 9.1 & 23.9 & 26.3 & 20.6 & 19.3 & 380.5 & \begin{tabular}{|l|}
384.0 \\
\end{tabular} & 8.0 & 8.6 & 1.30 & 1.36 \\
\hline 35 & $8-2-1-10$ & 4.5 & 4.1 & 3.8 & 4.0 & 17.4 & 16.3 & 1.50 & 1.52 & 6.4 & 6.4 & \begin{tabular}{|l|}
32.1 \\
\end{tabular} & 34.5 & 24.2 & 23.7 & 295.5 & \begin{tabular}{|l|}
291.0 \\
\end{tabular} & \begin{tabular}{|l|}
9.4 \\
\end{tabular} & 9.9 & 1.40 & $\mid$\begin{tabular}{|l|} 
\\
\end{tabular} \\
\hline 36 & $8-2-1-12$ & 5.4 & 5.8 & 7.5 & 7.3 & 40.5 & 42.5 & 0.90 & 0.88 & 11.7 & 11.5 & 40.0 & 41.5 & 32.3 & 35.6 & 188.0 & $\mid 184.0$ & 5.3 & 4.8 & 1.20 & 1.17 \\
\hline 37 & $8-2-1-3$ & 3.1 & 3.0 & 4.9 & 5.1 & 17.4 & $\mid 15.1$ & 1.20 & 1.17 & 5.8 & 5.8 & \begin{tabular}{|l|}
35.0 \\
\end{tabular} & 37.4 & 32.7 & 31.4 & 238.5 & 232.5 & 5.6 & 5.1 & 1.13 & $\mid 1.19$ \\
\hline 38 & $8-2-1-6$ & 4.9 & 4.3 & 3.1 & 3.1 & 16.2 & 13.8 & 1.70 & 1.68 & 9.1 & 9.1 & 24.9 & 29.8 & 18.2 & 21.0 & 430.5 & 434.0 & 11.4 & 11.9 & 1.40 & $\mid 1.42$ \\
\hline 39 & $8-2-1-9$ & 5.9 & 5.4 & 2.7 & 2.9 & 16.0 & 15.5 & 0.90 & 0.87 & 10.7 & 10.5 & 26.0 & 28.4 & 21.7 & 20.4 & 337.8 & 331.8 & 8.6 & 9.2 & 1.30 & $\mid 1.39$ \\
\hline 40 & $7-5-3-5$ & 4.2 & 3.7 & 2.8 & 2.9 & 11.6 & 10.4 & 0.80 & 0.82 & 9.6 & 9.6 & \begin{tabular}{|l|}
26.9 \\
\end{tabular} & 29.3 & 20.5 & \begin{tabular}{|l|}
20.8 \\
\end{tabular} & 339.5 & 330.0 & 6.2 & 6.6 & 1.37 & $\mid 1.41$ \\
\hline 41 & $\begin{array}{c}\text { Philippine } \\
\text { 28-2 }\end{array}$ & 3.8 & 3.2 & 2.1 & 2.3 & 8.0 & 7.3 & 0.40 & 0.34 & 3.0 & 3.0 & 23.4 & 22.8 & 18.5 & 17.2 & 119.0 & $\mid 114.5$ & 14.9 & 15.2 & 1.30 & 1.33 \\
\hline 42 & $\begin{array}{c}\text { Philippine } \\
\text { 25-3 }\end{array}$ & 3.8 & 3.3 & 3.8 & 3.6 & 14.1 & 12.0 & 1.60 & 1.57 & 5.0 & 5.0 & 28.7 & 32.7 & 25.9 & 23.1 & 288.0 & 282.0 & 8.3 & 7.8 & 1.27 & $\mid 1.42$ \\
\hline & Mean & 4.5 & 4.3 & 4.1 & 4.2 & 18.5 & 17.9 & 1.24 & 1.22 & 8.6 & 8.6 & 31.7 & 33.2 & 26.5 & 25.6 & 269.0 & \begin{tabular}{|l|}
266.4 \\
\end{tabular} & 8.0 & 8.2 & 1.27 & 1.32 \\
\hline & Range & $\begin{array}{c}2.9 \text { to } \\
7\end{array}$ & \begin{tabular}{|c|}
2.8 to \\
6.6
\end{tabular} & \begin{tabular}{|l|}
1.9 to \\
8.2
\end{tabular} & $\begin{array}{l}2 \text { to } \\
8.1\end{array}$ & $\begin{array}{c}8.1 \text { to } \\
40.5\end{array}$ & $\begin{array}{c}7.3 \text { to } \\
42.5\end{array}$ & 0.4 to 2 & $\begin{array}{c}0.34 \text { to } \\
1.98\end{array}$ & $\begin{array}{l}3 \text { to } \\
12.3\end{array}$ & \begin{tabular}{|l}
3 to \\
12.4
\end{tabular} & \begin{tabular}{|c|}
18.8 to \\
43.7
\end{tabular} & $\begin{array}{c}17.7 \text { to } \\
42.1\end{array}$ & $\begin{array}{c}12.6 \text { to } \\
39.5\end{array}$ & \begin{tabular}{|c|}
13 to \\
38.2
\end{tabular} & $\begin{array}{c}35.8 \text { to } \\
435.5\end{array}$ & \begin{tabular}{|c|}
37.8 to \\
434
\end{tabular} & $\begin{array}{l}3 \text { to } \\
14.9\end{array}$ & \begin{tabular}{|c|}
3.1 to \\
15.2
\end{tabular} & \begin{tabular}{|c|}
1.07 to \\
1.47
\end{tabular} & \begin{tabular}{|c|}
1.1 to \\
1.44
\end{tabular} \\
\hline & LSD & 4.5 & 4.3 & 4.1 & 4.2 & 18.5 & 17.9 & 1.24 & 1.22 & 8.6 & 8.6 & 31.7 & 33.2 & 26.5 & 25.6 & 269.0 & \begin{tabular}{|l|}
266.4 \\
\end{tabular} & 8.0 & 8.2 & 1.27 & 1.32 \\
\hline
\end{tabular}




\section{8- Number of seeds per fruit}

Number of seeds per fruit of the studied inbred lines had wide rang from 35.8 to 435.5 seeds (Table 2 ) in both seasons. The genotypes 2-3-4-11 and 6-2-3-16 had the lowest number of seeds per fruit with significant differences with the other inbred lines in 2016 and 2017. on the other hand, 2-3-1-2 and 82-1-6 had the highest number of seeds per fruit with significant differences with the other inbred lines in 2016 and 2017. Anburani et al (2019) studied thirty watermelon genotypes and found that number of seeds per fruit ranged from 115.00 to 422.67 seeds. Messiaen (1994) reported that watermelon seeds are relatively large, with 7-20 seeds/g, varying in color i.e., white, green, yellow, grey, tan, brown, red, black as well as mixed colours. Seeds continue to mature as the fruit ripens and the rind lightens in color.

\section{9- 100 seeds weight (g):}

100 seeds weight of the studied inbred lines had wide rang from 3 to $15.2 \mathrm{~g}$ (Table 2) in both seasons. The inbred lines 2-4-1-1and 6-2-3-2 had the lightest 100 seed weight with significant differences with the other inbred lines in 2016 and 2017. on the other hand, 2-2-1-2 and Philippine 28-2 had the heaviest 100 seeds weight with significant differences with the other inbred lines in 2016 and 2017. Anburani et al (2019) studied thirty watermelon genotypes and found that 100 seed weight ranged between 4.25 and $12.63 \mathrm{~g}$.

\section{0-Fruit shape index}

Data obtained on fruit shape index trait are presented in table (2). The results showed that the values of fruit shape index ranged from 1.07 to 1.43 in the both seasons, these values referred that the shape of all inbred lines were round and cylindrical, this scale of fruit shape index of melon fruits by Rashidi and Seyfi (2007). These shapes are excellent for market. Gusmini and Wehner (2005) studied the length/diameter ratio (L/D) in watermelon and found that fruit shape index ranged between 1.1 and 2.3.

These results agreement with Jelica et al (2011) which reported that the current demands of both consumers and producers are an important item in watermelon breeding programs, especially for fruit traits and said these requirements are: fruit size of $4-6 \mathrm{~kg}$, good taste $(8-10 \%$ SS), rind thickness up to $1.5 \mathrm{~cm}$, round to oval-shaped fruit, small seeds and very high meat ratio.

\section{Genetic studies}

Selection is the fundamental process in the development of superior varieties and it depends on the variability available in the crop. Genetic variability is the basic need for a plant breeder to initiate any breeding programme. A wide range was observed for the major yield contributing characters and wide variances were observed for these traits.

\section{- PCV and GCV}

Greater variability ensures better changes of producing new desirable forms. Phenotypic coefficient of variation (PCV) and genotypic coefficient of variation (GCV) were computed for ten studied characters on 42 genotypes of 
watermelon (Table 3). The PCV for different characters ranged from $14.86 \%$ (fruit shape index) to $73.84 \%$ (total yield per plant). The GCV ranged from $14.05 \%$ (fruit shape index) to $72.98 \%$ (total yield per plant). High amount of fixable variation in watermelon characters has been reported by Lalta $\boldsymbol{e t}$ al. (1998). In the present study, high estimates of both GCV and PCV were registered for the studied traits, viz., FW (60.21 and 60.31\% respectively), NS (66.04 and 66.06\% respectively), SW (61.84 and 62.12\% respectively) and TY (72.98 and $73.84 \%$ respectively) which suggest greater phenotypic and genotypic variability among the accession and responsiveness of the attributes for making further improvement by selection.

Moderate estimates of GCV and PCV were registered for the traits FD (40.13and $40.13 \%$ respectively), TSS (46.43 and $46.45 \%$ respectively), NF (39.18 and $40.31 \%$ respectively) and RT (51.27 and 51.32\% respectively).

Low estimates of GCV and PCV were registered for the traits viz., FL (29.14and 29.79\% respectively) and SHI (14.05and 14.86\% respectively), which indicated that these traits were less affected by environment. (Indiresh 1982) reported similar results in bitter gourd. This suggests that these characters may be less influenced by environment which is agreement with the findings of Tomer et al. (2008) in muskmelon.

Table 3. Estimation of PCV, GCV, heritability and genetic advance as per cent of mean for various characters in watermelon.

\begin{tabular}{|c|c|c|c|c|c|c|c|}
\hline No. & Characters & $\mathbf{V}_{\mathbf{E}}$ & $\mathbf{V}_{\mathbf{G}}$ & $\mathbf{V}_{\mathbf{P}}$ & $\mathbf{G C V}$ & PCV & $\mathbf{h}^{2}$ \\
\hline $\mathbf{1}$ & FD & $\mathbf{0 . 9 9}$ & $\mathbf{1 0 5 . 3 5}$ & $\mathbf{1 0 6 . 3 4}$ & $\mathbf{4 0 . 1 3}$ & $\mathbf{4 0 . 3 1}$ & $\mathbf{9 9 . 0 7}$ \\
\hline $\mathbf{2}$ & FL & 4.23 & $\mathbf{9 3 . 4 6}$ & $\mathbf{9 7 . 6 9}$ & $\mathbf{2 9 . 1 4}$ & $\mathbf{2 9 . 7 9}$ & $\mathbf{9 5 . 6 7}$ \\
\hline 3 & FW & $\mathbf{0 . 0 2}$ & $\mathbf{6 . 2 7}$ & $\mathbf{6 . 2 8}$ & $\mathbf{6 0 . 2 1}$ & $\mathbf{6 0 . 3 1}$ & $\mathbf{9 9 . 6 9}$ \\
\hline 4 & NF & $\mathbf{0 . 1 6}$ & $\mathbf{2 . 8 0}$ & $\mathbf{2 . 9 7}$ & $\mathbf{3 9 . 1 8}$ & $\mathbf{4 0 . 3 1}$ & $\mathbf{9 4 . 4 5}$ \\
\hline $\mathbf{5}$ & NS & $\mathbf{1 7 . 3 0}$ & $\mathbf{3 0 9 5 6 . 4 0}$ & $\mathbf{3 0 9 7 3 . 7 0}$ & $\mathbf{6 6 . 0 4}$ & $\mathbf{6 6 . 0 6}$ & $\mathbf{9 9 . 9 4}$ \\
\hline $\mathbf{6}$ & SW & $\mathbf{0 . 2 3}$ & $\mathbf{2 5 . 4 9}$ & $\mathbf{2 5 . 7 2}$ & $\mathbf{6 1 . 8 4}$ & $\mathbf{6 2 . 1 2}$ & $\mathbf{9 9 . 1 1}$ \\
\hline 7 & TSS & $\mathbf{0 . 0 2}$ & $\mathbf{1 6 . 3 0}$ & $\mathbf{1 6 . 3 1}$ & $\mathbf{4 6 . 4 3}$ & $\mathbf{4 6 . 4 5}$ & $\mathbf{9 9 . 9 0}$ \\
\hline $\mathbf{8}$ & TY & $\mathbf{4 . 0 5}$ & $\mathbf{1 6 9 . 9 7}$ & $\mathbf{1 7 4 . 0 2}$ & $\mathbf{7 2 . 9 8}$ & $\mathbf{7 3 . 8 4}$ & $\mathbf{9 7 . 6 7}$ \\
\hline $\mathbf{9}$ & RT & $\mathbf{0 . 0 0}$ & $\mathbf{0 . 3 9}$ & $\mathbf{0 . 3 9}$ & $\mathbf{5 1 . 2 7}$ & $\mathbf{5 1 . 3 2}$ & $\mathbf{9 9 . 8 2}$ \\
\hline 10 & SHI & $\mathbf{0 . 0 0 4}$ & $\mathbf{0 . 0 3 2}$ & $\mathbf{0 . 0 4}$ & $\mathbf{1 4 . 0 5}$ & $\mathbf{1 4 . 8 6}$ & $\mathbf{8 9 . 3 6}$ \\
\hline
\end{tabular}

$\mathrm{NF}=$ Number of fruits per plant, $\mathrm{FW}=$ fruit weight $(\mathrm{kg}), \mathrm{TY}=$ Total yield per plant $(\mathrm{kg}), \mathrm{RT}=\mathrm{Rind}$ thickness TSS $=$ Total soluble solids $(\%)$, FL=Fruit length $(\mathrm{cm})$, FD=Fruit diameter $(\mathrm{cm})$, SHI = shape indexNS= Number of seeds per fruit and $\mathrm{SW}=100$ seed weight $(\mathrm{g})$

\section{- Heritability}

In the present study, almost all the characters exhibited high heritability value which ranged from 89.36 to 99.94 per cent. The characters included 100 seed weight, number of seed per fruit, single fruit weight, fruit diameter, fruit length, flesh thickness, and yield per plant. High heritability values obtained for all traits in the present study suggest that these traits may generally be governed by additive gene action and hence the phenotype would provide a fairly reliable measure of the genotype which provides scope for selection based on the phenotypic performance.

In general, we can notice that the differences between phenotypic and genotypic variance for all studied traits were low. In other words, the 
large portion of phenotypic variance $\left(\sigma_{p}^{2}\right)$ was due to the genetic variance $\left(\sigma_{\mathrm{g}}^{2}\right)$. Consequently, estimated broad-sense heritability showed high values for these traits, indicating that the observed significant phenotypic differences among the studied inbred lines are of genetic nature and there are small environmental effects on the phenotypic variation. Therefore, these characters can be improved through selection based on phenotypic observations in early segregating generations in watermelon germplasm.

\section{- Correlation}

Simple correlation coefficient among 42 watermelon inbred lines is presented in (Table 4). The result indicated highly significant positive correlation for FD with FL (0.66), FW(0.23) and TY (0.44). Whereas negative correlation was recorded with SHI(-0.26). Significant and highly positive correlation for FL with FW (0.33) and TY (0.50) respectively were also observed. Whereas negative correlation was recorded with SHI (-0.12). Significant and highly positive correlation for FW with RT (0.15) and TY (0.82) respectively were also observed. Whereas negative correlation was recorded with SHI(-0.31).Significant and highly positive correlation for NF with TSS $(0.51)$ and TY $(0.67)$ respectively were also observed. Whereas negative correlation was recorded with SHI (-0.09).Significant and highly negative correlation was recorded for TY with SHI -0.27).

Table 4: Simple correlations coefficient among 42 watermelon inbred lines.

\begin{tabular}{|c|c|c|c|c|c|c|c|c|c|}
\hline & FD & FL & FW & NF & NS & RT & SW & TSS & TY \\
\hline FL & $\mathbf{0 . 6 6}$ & & & & & & & & \\
\hline FW & $\mathbf{0 . 2 3}$ & $\mathbf{0 . 3 3}$ & & & & & & & \\
\hline NF & $\mathbf{0 . 4 5}$ & $\mathbf{0 . 3 8}$ & $\mathbf{0 . 1 6}$ & & & & & & \\
\hline NS & $-\mathbf{- 0 . 3 2}$ & $\mathbf{- 0 . 4 7}$ & $\mathbf{- 0 . 2 1}$ & $-\mathbf{0 . 2 2}$ & & & & & \\
\hline RT & $\mathbf{- 0 . 3 4}$ & $\mathbf{- 0 . 2 2}$ & $\mathbf{0 . 1 5}$ & $-\mathbf{0 . 2 7}$ & $\mathbf{0 . 4 0}$ & & & & \\
\hline SW & $\mathbf{0 . 0 4}$ & $\mathbf{- 0 . 1 8}$ & $\mathbf{- 0 . 4 9}$ & $\mathbf{- 0 . 1 6}$ & $\mathbf{0 . 0 5}$ & $\mathbf{- 0 . 1 5}$ & & & \\
\hline TSS & $\mathbf{0 . 3 9}$ & $\mathbf{0 . 1 3}$ & $\mathbf{0 . 2 2}$ & $\mathbf{0 . 5 1}$ & $\mathbf{0 . 2 8}$ & $\mathbf{0 . 0 2}$ & $\mathbf{- 0 . 1 4}$ & & \\
\hline TY & $\mathbf{0 . 4 4}$ & $\mathbf{0 . 5 0}$ & $\mathbf{0 . 8 2}$ & $\mathbf{0 . 6 7}$ & $\mathbf{- 0 . 3 4}$ & $\mathbf{- 0 . 0 8}$ & $\mathbf{- 0 . 4 4}$ & $\mathbf{0 . 4 1}$ & \\
\hline SHI & $\mathbf{- 0 . 2 6}$ & $\mathbf{- 0 . 1 2}$ & $\mathbf{- 0 . 3 1}$ & $\mathbf{- 0 . 0 9}$ & $\mathbf{0 . 1 3}$ & $\mathbf{0 . 0 7}$ & $\mathbf{0 . 1 5}$ & $\mathbf{- 0 . 0 8}$ & $\mathbf{- 0 . 2 7}$ \\
\hline
\end{tabular}

$\mathrm{NF}=$ Number of fruits per plant, $\mathrm{FW}=$ fruit weight $(\mathrm{kg}), \mathrm{TY}=$ Total yield per plant $(\mathrm{kg})$, RT=Rind thickness TSS $=$ Total soluble solids $(\%)$, FL=Fruit length $(\mathrm{cm})$, FD=Fruit diameter $(\mathrm{cm}), \mathrm{SHI}=$ shape indexNS $=$ Number of seeds per fruit and $\mathrm{SW}=100$ seed weight (g)

\section{CONCLUSION}

The results can be concluded that variability studies showed significant differences among the 42 watermelon inbred lines for all the studied10 characters. 8-2-1-12 had the best marketable yield and fruit quality on shape (excellent round shape), fruit weight $(7.3$ and $7.5 \mathrm{~kg}$ ), 
number of fruits per plant $(7.5$ and $7.3 \mathrm{~cm})$, total yield per plant (above $40 \mathrm{~kg}$ ), TSS (11.7 and 11.5\%) and the flesh color is dark red, but the seeds is very big, so, this inbred line is suggested to be introduction in breeding programme for red $\mathrm{F}_{1}$ hybrids production.6-2-2-16, 6-2-3-15 and 6-2-3-16 are the best marketable yield and fruit quality as yellow watermelon inbred lines of shape, fruit weight, number of fruits per plant, total yield per plant, TSS and the flesh color, and they are suggested to be introduction in breeding programme for yellow $F_{1}$ hybrids production. Genetic analysis indicated maximum phenotypic and genotypic coefficient of variation for most characters. High heritability observed for all characters.

\section{REFERENCES}

Anburani, A. ; P. Kannan and K. Muthumanickam (2019). Genetic variability, heritability and genetic advance for yield and yield components in watermelon (Citrullus lanatus Thunb.). World News Natural Sciences, 25:22-30.

Bretting, P.K. and M.P. Widrlechner (1995). Genetic Markers and Plant Genetic Resource Management. In: Plant Breeding Reviews. (Ed.): J. Janick. John Wiley \& Sons, Inc., New York.13: 11-87.

Goreta, S. ; S. Perica ; G. Dumicic ; L. Bucan and K. Zanic (2005). Growth and yield of watermelon on polyethylene mulch with different spacing and nitrogen rates. Amer. J. Hort. Sci., 40 (2): 366-369.

Gusmini, G. and T. C. Wehner (2005). Rind thickness of watermelon cultivar for use in pickle production. Hor. Tehnology, 14 (4): 540-545.

Gusmini, G. ; T. C. Wehner and R. L. Jarret (2004). Inheritance of egusi type in watermelon. J. Heredity, 95: 268-270.

Indiresh, B. T. (1982). Studies on Genotypic Variability in Bitter Gourd (Momordicach arantia L.) M.Sc. (Agri.) Thesis abstr. 8 (1): Univ. Agri. Sci. Bangalore.

Jelica, G.V. ; M. Vasic ; D. Milic and J. Cervenski (2011). Diallel cross analysis for fruit traits in watermelon. Genetika, 43 (1): 163-174.

Johnson, H. W., H. F. Robinson and R. F. Comstock (1955). Estimates of genetic and environmental variability in soybean. Agronomy. J., 47: 314-318. 
Lalta, P. ; N. C. Gautam and S. P. Singh (1998). Studies on genetic variability and character association in watermelon (Citrullus lanatus (Thunb) Mansf). Veg. Sci., 15 (1): 86-94.

Messiaen, C. M. (1994). The Tropical Vegetable Garden, principles for improvement and increased production with application to the main vegetable types. The Macmillan Press Ltd. London.

Mujaju, C. (2009). Diversity of landraces and wild forms of watermelon (Citrullus lanatus) in southern Africa. Faculty of Landscape Planning, Horticulture and Agricultural Science.S wedish University of Agricultural Sciences. PhD, 40 p.

Rashidi, M. and K. Seyfi (2007). Classification of fruit shape in cantaloupe using the analysis of geometrical attributes. World J. Agric. Sci., 3 (6): 735-740.

Sivasubramanian S. and M. Menan (1973). Heterosis and inbreeding depression in rice. Madras Agric. J., 60: 1139.

Tomer, R.S. ; G.U. Kulkarni ; D.K. Kakade ; A.D. Patel and R. R. Acharya (2008). Genetic variability, correlation and path analysis in musk melon (Cucumis melo L.). Asian J. Hort., 3(1): $158-161$. 